\title{
The joint essential numerical range of operators: convexity and related results
}

\author{
by
}

Chi-Kwong Li (Williamsburg, VA) and Yiu-Tung Poon (Ames, IA)

\begin{abstract}
Let $W(\mathbf{A})$ and $W_{\mathrm{e}}(\mathbf{A})$ be the joint numerical range and the joint essential numerical range of an $m$-tuple of self-adjoint operators $\mathbf{A}=\left(A_{1}, \ldots, A_{m}\right)$ acting on an infinite-dimensional Hilbert space. It is shown that $W_{\mathrm{e}}(\mathbf{A})$ is always convex and admits many equivalent formulations. In particular, for any fixed $i \in\{1, \ldots, m\}, W_{\mathrm{e}}(\mathbf{A})$ can be obtained as the intersection of all sets of the form

$$
\operatorname{cl}\left(W\left(A_{1}, \ldots, A_{i+1}, A_{i}+F, A_{i+1}, \ldots, A_{m}\right)\right),
$$

where $F=F^{*}$ has finite rank. Moreover, the closure $\operatorname{cl}(W(\mathbf{A}))$ of $W(\mathbf{A})$ is always starshaped with the elements in $W_{\mathrm{e}}(\mathbf{A})$ as star centers. Although $\operatorname{cl}(W(\mathbf{A}))$ is usually not convex, an analog of the separation theorem is obtained, namely, for any element $\mathbf{d} \notin$ $\operatorname{cl}(W(\underset{\tilde{\mathbf{A}}}{\mathbf{A}}))$, there is a linear functional $f$ such that $f(\mathbf{d})>\sup \{f(\mathbf{a}): \mathbf{a} \in \operatorname{cl}(W(\tilde{\mathbf{A}}))\}$, where $\tilde{\mathbf{A}}$ is obtained from $\mathbf{A}$ by perturbing one of the components $A_{i}$ by a finite rank self-adjoint operator. Other results on $W(\mathbf{A})$ and $W_{\mathrm{e}}(\mathbf{A})$ extending those on a single operator are obtained.
\end{abstract}

1. Introduction. Let $\mathcal{B}(\mathcal{H})$ denote the algebra of bounded linear operators acting on a complex Hilbert space $\mathcal{H}$. The numerical range of $A \in \mathcal{B}(\mathcal{H})$ is defined as

$$
W(A)=\{\langle A \mathbf{x}, \mathbf{x}\rangle: \mathbf{x} \in \mathcal{H},\langle\mathbf{x}, \mathbf{x}\rangle=1\},
$$

which is useful in studying operators; see [10,11, 22, 24] and [25, Chapter 1]. Let $\mathcal{S}(\mathcal{H})$ denote the set of self-adjoint operators in $\mathcal{B}(\mathcal{H})$. Since every $A \in$ $\mathcal{B}(\mathcal{H})$ admits a decomposition $A=A_{1}+i A_{2}$ with $A_{1}, A_{2} \in \mathcal{S}(\mathcal{H})$, we can identify $W(A)$ with

$$
\left\{\left(\left\langle A_{1} \mathbf{x}, \mathbf{x}\right\rangle,\left\langle A_{2} \mathbf{x}, \mathbf{x}\right\rangle\right): \mathbf{x} \in \mathcal{H},\langle\mathbf{x}, \mathbf{x}\rangle=1\right\} \subseteq \mathbb{R}^{2} .
$$

This leads to the joint numerical range of $\mathbf{A}=\left(A_{1}, \ldots, A_{m}\right) \in \mathcal{S}(\mathcal{H})^{m}$,

$$
W(\mathbf{A})=\left\{\left(\left\langle A_{1} \mathbf{x}, \mathbf{x}\right\rangle, \ldots,\left\langle A_{m} \mathbf{x}, \mathbf{x}\right\rangle\right): \mathbf{x} \in \mathcal{H},\langle\mathbf{x}, \mathbf{x}\rangle=1\right\} \subseteq \mathbb{R}^{m}
$$

2000 Mathematics Subject Classification: 47A12, 47A13, 47A55.

Key words and phrases: joint essential numerical range, self-adjoint operator, Hilbert space. 
which has been studied by many researchers in order to understand the joint behavior of several operators $A_{1}, \ldots, A_{m}$. One may see $[1,5,12,14,15,16$, $19,23,28,31,33,35]$ and their references for the background and many applications of the joint numerical range.

Let $\mathcal{F}(\mathcal{H})$ and $\mathcal{K}(\mathcal{H})$ be the sets of finite rank and compact operators in $\mathcal{B}(\mathcal{H})$. In the study of finite rank or compact perturbations of operators, researchers consider the joint essential numerical range of $\mathbf{A} \in \mathcal{S}(\mathcal{H})^{m}$ defined by

$$
W_{\mathrm{e}}(\mathbf{A})=\bigcap\left\{\mathbf{c l}(W(\mathbf{A}+\mathbf{K})): \mathbf{K}=\left(K_{1}, \ldots, K_{m}\right) \in \mathcal{K}(\mathcal{H})^{m} \cap \mathcal{S}(\mathcal{H})^{m}\right\} .
$$

Here $\operatorname{cl}(S)$ denotes the closure of the set $S$. For $m=2, W_{\mathrm{e}}(\mathbf{A})$ can be identified with the essential numerical range of $A=A_{1}+i A_{2} \in \mathcal{B}(\mathcal{H})$, defined by

$$
W_{\mathrm{e}}(A)=\bigcap\{\operatorname{cl}(W(A+K)): K \in \mathcal{K}(\mathcal{H})\} .
$$

One may see $[2,3,6,7,13,18,20,21,26,27,30,32,36,37]$ for many interesting results on $W_{\mathrm{e}}(A)$ and $W_{\mathrm{e}}(\mathbf{A})$.

In theoretical studies as well as applications, it is desirable to deal with $\mathbf{A}$ such that $W(\mathbf{A})$ or $\mathbf{c l}(W(\mathbf{A}))$ is convex. For example, if $\mathbf{c l}(W(\mathbf{A}))$ is convex, one can apply the separation theorem to show that $\mathbf{0} \notin \mathbf{c l}(W(\mathbf{A}))$ if and only if there exist $r>0$ and $\mathbf{c}=\left(c_{1}, \ldots, c_{m}\right) \in \mathbb{R}^{m}$ such that $\left(\sum_{i=1}^{m} c_{i} A_{i}\right)>$ $r I_{\mathcal{H}}$. Unfortunately, $\operatorname{cl}(W(\mathbf{A}))$ is not always convex. Here are some results concerning the convexity of $W(\mathbf{A})$ and $\mathbf{c l}(W(\mathbf{A}))$, and related to $W_{\mathrm{e}}(\mathbf{A})$ (for example, see $[5,10,11,36,21,29,31]$ and their references).

(P1) [31] $W\left(A_{1}, \ldots, A_{m}\right)$ is convex if (a) $\operatorname{span}\left\{I, A_{1}, \ldots, A_{m}\right\}$ has dimension at most 3 , or (b) $\operatorname{dim} \mathcal{H} \geq 3$ and $\operatorname{span}\left\{I, A_{1}, \ldots, A_{m}\right\}$ has dimension at most 4 .

(P2) [31] For any $A_{1}, A_{2}, A_{3} \in \mathcal{S}(\mathcal{H})$ such that $\operatorname{span}\left\{I, A_{1}, A_{2}, A_{3}\right\}$ has dimension 4 , there is always an $A_{4} \in \mathcal{S}(\mathcal{H})$ for which $W\left(A_{1}, \ldots, A_{4}\right)$ is not convex.

(P3) [31] If $m \geq 4$ then there exists $\mathbf{A} \in \mathcal{S}(\mathcal{H})^{m}$ such that $W(\mathbf{A})$ is non-convex.

(P4) For any positive integer $m$ and any $\mathbf{A} \in \mathcal{S}(\mathcal{H})^{m}, W_{\mathrm{e}}(\mathbf{A})$ is a compact set contained in $W(\mathbf{A})$. If $\operatorname{span}\left\{I, A_{1}, \ldots, A_{m}\right\}$ has dimension at most 4 , then $W_{\mathrm{e}}(\mathbf{A})$ is convex.

(P5) [36] For $S \subseteq \mathbb{R}^{m}$, let $\operatorname{Ext}(S)$ be the set of all points in $S$ that do not lie in the open line segment joining two distinct points in $S$. Then $\operatorname{Ext}(\mathbf{c l}(W(\mathbf{A}))) \subseteq \operatorname{Ext}(W(\mathbf{A})) \cup \operatorname{Ext}\left(W_{\mathrm{e}}(\mathbf{A})\right)$.

We remark that $(\mathrm{P} 1)-(\mathrm{P} 3)$ also hold if we replace $W(\mathbf{A})$ by $\operatorname{cl}(W(\mathbf{A}))$. In view of (P2) and (P3), if $m>3$, then for $\mathbf{A} \in \mathcal{S}(\mathcal{H})^{m}$ and $\mathbf{K} \in$ $\mathcal{K}(\mathcal{H})^{m} \cap \mathcal{S}(\mathcal{H})^{m}$ the set $\mathbf{c l}(W(\mathbf{A}+\mathbf{K}))$ is usually non-convex. Since $W_{\mathrm{e}}(\mathbf{A})$ 
is the intersection of non-convex sets, one does not expect the set $W_{\mathrm{e}}(\mathbf{A})$ to be convex. This might be the reason why the convexity of $W_{\mathrm{e}}(\mathbf{A})$ is seldom discussed for $m>3$. In fact, some researchers have studied different geometrical properties of $W_{\mathrm{e}}(\mathbf{A})$ under the assumption that $W_{\mathrm{e}}(\mathbf{A})$ is convex, and some have examined $W_{\mathrm{e}}(\mathbf{A})$ for different classes of operators without discussing their convexity; for example, see [6, 26, 27, 30, 32].

In this paper, we prove the rather unexpected result that $W_{\mathrm{e}}(\mathbf{A})$ is always convex. Moreover, it is shown that the closure $\operatorname{cl}(W(\mathbf{A}))$ of $W(\mathbf{A})$ is always star-shaped with the elements in $W_{\mathrm{e}}(\mathbf{A})$ as star centers. Many results relating $W_{\mathrm{e}}(\mathbf{A})$ and $W(\mathbf{A})$ are also obtained. Our paper is organized as follows.

In Section 2, we extend the results of [21] by establishing several equivalent formulations of the essential joint numerical range for $\mathbf{A} \in \mathcal{S}(\mathcal{H})^{m}$. One key obstacle for such an extension is the fact that $W(\mathbf{A})$ may not be convex. To get around this problem, we show that $\mathbf{c l}(W(\mathbf{A}))$ is star-shaped. The star-shapedness of $\mathbf{c l}(W(\mathbf{A}))$ and the conditions equivalent to membership in $W_{\mathrm{e}}(\mathbf{A})$, given in Section 2, lead to our main result that $W_{\mathrm{e}}(\mathbf{A})$ is convex and its elements are star centers of the $\operatorname{set} \operatorname{cl}(W(\mathbf{A}))$, which is presented in Section 3. With the convexity theorem, we obtain additional descriptions of $W_{\mathrm{e}}(\mathbf{A})$ in Section 4 in terms of the perturbations of one of the components of $\mathbf{A}$, and also in terms of linear combinations of the components of $\mathbf{A}$. For example, we show that $W_{\mathrm{e}}\left(A_{1}, \ldots, A_{m}\right)$ is equal to the sets

$$
\bigcap\left\{\mathbf{c l}\left(W\left(A_{1}, \ldots, A_{i-1}, A_{i}+F, A_{i+1}, \ldots, A_{m}\right): F \in \mathcal{F}(\mathcal{H}) \cap \mathcal{S}(\mathcal{H})\right\}\right.
$$

and

$$
\left\{\left(a_{1}, \ldots, a_{m}\right): \sum_{j=1}^{m} c_{j} a_{j} \in W_{\mathrm{e}}\left(\sum_{j=1}^{m} c_{j} A_{j}\right) \text { for all }\left(c_{1}, \ldots, c_{m}\right) \in \Omega\right\},
$$

where $\Omega=\left\{\left(c_{1}, \ldots, c_{m}\right) \in \mathbb{R}^{m}: \sum_{j=1}^{m} c_{j}^{2}=1\right\}$. Also, we obtain an ana$\log$ of the separation theorem for the not necessarily convex set $\operatorname{cl}(W(\mathbf{A}))$, namely, for any element $\mathbf{d} \notin \mathbf{c l}(W(\mathbf{A}))$, there is a linear functional $f$ such that $f(\mathbf{d})>\sup \{f(\mathbf{a}): \mathbf{a} \in \mathbf{c l}(W(\tilde{\mathbf{A}}))\}$, where $\tilde{\mathbf{A}}$ is obtained from $\mathbf{A}$ by perturbing one of the components $A_{j}$ by a finite rank self-adjoint operator. In Section 5, we present additional results on $W(\mathbf{A})$ and $W_{\mathrm{e}}(\mathbf{A})$. For instance, $W_{\mathrm{e}}(\mathbf{A})=\mathbf{c l}(W(\mathbf{A}))$ if and only if the extreme points of $W(\mathbf{A})$ are contained in $W_{\mathrm{e}}(\mathbf{A})$; the convex hull of $\mathbf{c l}(W(\mathbf{A}))$ can always be realized as the joint essential numerical range of $\left(\tilde{A}_{1}, \ldots, \tilde{A}_{m}\right)$ for linear operators $\tilde{A}_{1}, \ldots, \tilde{A}_{m}$ acting on a separable Hilbert space.

In our discussion, we always assume that $\mathcal{H}$ is infinite-dimensional. For any vector $\mathbf{x} \in \mathcal{H}$ and $\mathbf{A}=\left(A_{1}, \ldots, A_{m}\right) \in \mathcal{S}(\mathcal{H})^{m}$, we will use the notation

$$
\langle\mathbf{A x}, \mathbf{x}\rangle=\left(\left\langle A_{1} \mathbf{x}, \mathbf{x}\right\rangle, \ldots,\left\langle A_{m} \mathbf{x}, \mathbf{x}\right\rangle\right) .
$$


Furthermore, $\mathbb{R}^{m}$ will be used to denote the inner product space of $1 \times m$ real vectors with the usual inner product $\langle\mathbf{x}, \mathbf{y}\rangle$.

2. Equivalent conditions for $W_{\mathrm{e}}(\mathbf{A})$. Following [21, Theorem 5.1] and its corollary on a single operator $A \in \mathcal{B}(\mathcal{H})$, we obtain several conditions equivalent to membership in $W_{\mathrm{e}}(\mathbf{A})$.

Theorem 2.1. Let $\mathbf{A}=\left(A_{1}, \ldots, A_{m}\right) \in \mathcal{S}(\mathcal{H})^{m}$. The following conditions are equivalent for a real vector $\mathbf{a}=\left(a_{1}, \ldots, a_{m}\right)$ :

(1) $\mathbf{a} \in W_{\mathrm{e}}(\mathbf{A})=\bigcap\left\{\mathbf{c l}(W(\mathbf{A}+\mathbf{K})): \mathbf{K} \in \mathcal{K}(\mathcal{H})^{m} \cap \mathcal{S}(\mathcal{H})^{m}\right\}$.

(2) $\mathbf{a} \in \bigcap\left\{\mathbf{c l}(W(\mathbf{A}+\mathbf{F})): \mathbf{F} \in \mathcal{F}(\mathcal{H})^{m} \cap \mathcal{S}(\mathcal{H})^{m}\right\}$.

(3) There is an orthonormal sequence $\left\{\mathbf{x}_{n}\right\}_{n=1}^{\infty} \subset \mathcal{H}$ of vectors such that

$$
\lim _{n \rightarrow \infty}\left\langle\mathbf{A} \mathbf{x}_{n}, \mathbf{x}_{n}\right\rangle=\mathbf{a}
$$

(4) There is a sequence $\left\{\mathbf{x}_{n}\right\}_{n=1}^{\infty} \subset \mathcal{H}$ of unit vectors converging weakly to $\mathbf{0}$ in $\mathcal{H}$ such that

$$
\lim _{n \rightarrow \infty}\left\langle\mathbf{A} \mathbf{x}_{n}, \mathbf{x}_{n}\right\rangle=\mathbf{a} .
$$

(5) There is an infinite-dimensional projection $P \in \mathcal{S}(\mathcal{H})$ such that

$$
P\left(A_{j}-a_{j} I\right) P \in \mathcal{K}(\mathcal{H}) \quad \text { for } j=1, \ldots, k .
$$

Most of the argument in [21] can be applied here except for one crucial step, where the convexity of $W(\mathbf{A})$ for $m=2$ is needed. Since $W(\mathbf{A})$ may not be convex for $m>3$, we need the following auxiliary result to overcome the obstacle. As a byproduct, it shows that $\mathbf{c l}(W(\mathbf{A}))$ is star-shaped.

TheOREM 2.2. Let A satisfy the hypothesis of Theorem 2.1, and let $W_{3}(\mathbf{A})$ be the set of real vectors a satisfying condition (3) of Theorem 2.1. Then $W_{3}(\mathbf{A})$ is non-empty and closed. Moreover, each element $\mathbf{a} \in W_{3}(\mathbf{A})$ is a star center of $\mathbf{c l}(W(\mathbf{A}))$, i.e., for any $\mathbf{b} \in \mathbf{c l}(W(\mathbf{A}))$ we have $(1-t) \mathbf{a}+t \mathbf{b} \in$ $\operatorname{cl}(W(\mathbf{A}))$ for all $0 \leq t \leq 1$.

Proof. To prove that $W_{3}(\mathbf{A})$ is non-empty, let $\left\{\mathbf{x}_{n}\right\}_{n=1}^{\infty}$ be an orthonormal sequence of vectors in $\mathcal{H}$. Then the sequence $\left\{\left\langle\mathbf{A} \mathbf{x}_{n}, \mathbf{x}_{n}\right\rangle\right\}_{n=1}^{\infty}$ is bounded. By choosing a subsequence if necessary, we can assume that $\left\langle\mathbf{A} \mathbf{x}_{n}, \mathbf{x}_{n}\right\rangle$ converges. Hence, $W_{3}(\mathbf{A})$ is non-empty.

Next, we show that $W_{3}(\mathbf{A})$ is closed. Suppose $\mathbf{a} \in \mathbf{c l}\left(W_{3}(\mathbf{A})\right)$. Then for each $n \geq 1$, there exists an orthonormal sequence $\left\{\mathbf{x}_{k}^{n}\right\}_{k=1}^{\infty}$ such that

$$
\lim _{k \rightarrow \infty}\left\langle\mathbf{A} \mathbf{x}_{k}^{n}, \mathbf{x}_{k}^{n}\right\rangle=\mathbf{a}^{n} \in \mathbb{R}^{m} \text { and } \lim _{n \rightarrow \infty} \mathbf{a}^{n}=\mathbf{a} .
$$

Let $\delta_{n}=1 /\left(4 n^{2}\right)$. By going to subsequences if necessary, we may assume that $\left\|\left\langle\mathbf{A} \mathbf{x}_{k}^{n}, \mathbf{x}_{k}^{n}\right\rangle-\mathbf{a}\right\|<\delta_{n}$ for all $n, k$. We may also assume that $\left\|A_{1}\right\|^{2}+$ $\cdots+\left\|A_{m}\right\|^{2} \leq 1$. Then $\|\langle\mathbf{A x}, \mathbf{y}\rangle\| \leq\|\mathbf{x}\|\|\mathbf{y}\|$ for all $\mathbf{x}, \mathbf{y} \in \mathcal{H}$. 
Choose $\mathbf{x}_{1}=\mathbf{x}_{1}^{1}$. Then $\left\|\left\langle\mathbf{A} \mathbf{x}_{1}, \mathbf{x}_{1}\right\rangle-\mathbf{a}\right\|<1$. Suppose we have chosen $\left\{\mathbf{x}_{1}, \ldots, \mathbf{x}_{n}\right\}$ orthonormal with $\left\|\left\langle\mathbf{A x}_{k}, \mathbf{x}_{k}\right\rangle-\mathbf{a}\right\|<1 / k$ for $1 \leq k \leq n$. Then choose $N$ such that for all $1 \leq k \leq n$,

$$
\left|\left\langle\mathbf{x}_{k}, \mathbf{x}_{N}^{n+1}\right\rangle\right|,\left\|\left\langle\mathbf{A} \mathbf{x}_{k}, \mathbf{x}_{N}^{n+1}\right\rangle\right\|<\delta_{n+1} .
$$

Let $\mathbf{y}=\mathbf{x}_{N}^{n+1}-\sum_{k=1}^{n}\left\langle\mathbf{x}_{N}^{n+1}, \mathbf{x}_{k}\right\rangle \mathbf{x}_{k}$. Then

$$
\left\|\mathbf{y}-\mathbf{x}_{N}^{n+1}\right\| \leq n \delta_{n+1}, \quad \text { so } \quad 1-n \delta_{n+1} \leq\|\mathbf{y}\| \leq 1+n \delta_{n+1} .
$$

Therefore,

$$
\begin{aligned}
& \|\langle\mathbf{A y}, \mathbf{y}\rangle-\mathbf{a}\| \\
& \quad \leq\left\|\left\langle\mathbf{A}\left(\mathbf{y}-\mathbf{x}_{N}^{n+1}\right), \mathbf{y}\right\rangle\right\|+\left\|\left\langle\mathbf{A} \mathbf{x}_{N}^{n+1}, \mathbf{y}-\mathbf{x}_{N}^{n+1}\right\rangle\right\|+\left\|\left\langle\mathbf{A} \mathbf{x}_{N}^{n+1}, \mathbf{x}_{N}^{n+1}\right\rangle-\mathbf{a}\right\| \\
& \quad \leq\left\|\mathbf{y}-\mathbf{x}_{N}^{n+1}\right\|\left(\|\mathbf{y}\|+\left\|\mathbf{x}_{N}^{n+1}\right\|\right)+\delta_{n+1} \leq(2 n+2) \delta_{n+1} .
\end{aligned}
$$

Let $\mathbf{x}_{n+1}=\mathbf{y} /\|\mathbf{y}\|$. Then

$$
\left\|\mathbf{x}_{n+1}-\mathbf{y}\right\|=|1-\|\mathbf{y}\|| \leq n \delta_{n+1} .
$$

Hence, $\left\{\mathbf{x}_{1}, \ldots, \mathbf{x}_{n}, \mathbf{x}_{n+1}\right\}$ is an orthonormal set and

$$
\begin{aligned}
\left\|\left\langle\mathbf{A} \mathbf{x}_{n+1}, \mathbf{x}_{n+1}\right\rangle-\mathbf{a}\right\| & \leq\left\|\mathbf{y}-\mathbf{x}_{n+1}\right\|\left(\|\mathbf{y}\|+\left\|\mathbf{x}_{n+1}\right\|\right)+(2 n+2) \delta_{n+1} \\
& \leq(4 n+3) \delta_{n+1}<1 /(n+1) .
\end{aligned}
$$

To prove the last assertion, let $\mathbf{a} \in W_{3}(\mathbf{A})$ and $\mathbf{b} \in \mathbf{c l}(W(\mathbf{A}))$. Suppose $\left\{\mathbf{x}_{n}\right\}$ is an orthonormal sequence in $\mathcal{H}$ such that $\left\langle A \mathbf{x}_{n}, \mathbf{x}_{n}\right\rangle \rightarrow \mathbf{a}$. For $0 \leq$ $t \leq 1$, we are going to show that $(1-t) \mathbf{a}+t \mathbf{b} \in \mathbf{c l}(W(\mathbf{A}))$. Given $\varepsilon>0$, let $\mathbf{y}$ be a unit vector in $\mathcal{H}$ such that $\|\langle\mathbf{A y}, \mathbf{y}\rangle-\mathbf{b}\|<\varepsilon$. Choose $n$ such that $\left\|\left\langle\mathbf{A} \mathbf{x}_{n}, \mathbf{x}_{n}\right\rangle-\mathbf{a}\right\|<\varepsilon$ and $\left\|\left\langle\mathbf{A y}, \mathbf{x}_{n}\right\rangle\right\|<\varepsilon$. Choose $\theta \in \mathbb{R}$ such that $\left\langle e^{i \theta} \mathbf{y}, \mathbf{x}_{n}\right\rangle$ is imaginary. Let $\mathbf{z}=\sqrt{t} e^{i \theta} \mathbf{y}+\sqrt{1-t} \mathbf{x}_{n}$ Then

$$
\langle\mathbf{z}, \mathbf{z}\rangle=t\langle\mathbf{y}, \mathbf{y}\rangle+(1-t)\left\langle\mathbf{x}_{n}, \mathbf{x}_{n}\right\rangle+2 \sqrt{t} \sqrt{1-t}\left(\left\langle e^{i \theta} \mathbf{y}, \mathbf{x}_{n}\right\rangle+\left\langle\mathbf{x}_{n}, e^{i \theta} \mathbf{y}\right\rangle\right)=1
$$

and

$$
\begin{aligned}
\|\langle\mathbf{A z}, \mathbf{z}\rangle-((1-t) \mathbf{a}+t \mathbf{b})\| \leq & (1-t)\left\|\left\langle\mathbf{A} \mathbf{x}_{n}, \mathbf{x}_{n}\right\rangle-\mathbf{a}\right\|+t\|\langle\mathbf{A y}, \mathbf{y}\rangle-\mathbf{b}\| \\
& +\sqrt{t} \sqrt{1-t}\left\|\left\langle e^{i \theta} \mathbf{A y}, \mathbf{x}_{n}\right\rangle+\left\langle\mathbf{A} \mathbf{x}_{n}, e^{i \theta} \mathbf{y}\right\rangle\right\| \leq 2 \varepsilon .
\end{aligned}
$$

Therefore, $(1-t) \mathbf{a}+t \mathbf{b} \in \mathbf{c l}(W(\mathbf{A}))$.

The referee indicated that $W_{3}(\mathbf{A})$ is clearly closed, and a short proof is possible. We include a detailed proof for the sake of completeness and easy reference.

Proof of Theorem 2.1. For $j=2,3,4,5$, let $W_{j}(\mathbf{A})$ be the set of a satisfying condition $(j)$. Clearly, we have

$$
W_{5}(\mathbf{A}) \subseteq W_{3}(\mathbf{A}) \subseteq W_{4}(\mathbf{A}) \subseteq W_{\mathrm{e}}(\mathbf{A}) \subseteq W_{2}(\mathbf{A}) .
$$

Suppose $\mathbf{a} \in W_{2}(\mathbf{A})$. We are going to show that $\mathbf{a} \in W_{5}(\mathbf{A})$. Without loss of generality, we may assume $\mathbf{a}=\mathbf{0}$. 
Since $\mathbf{0} \in W_{2}(\mathbf{A}) \subseteq \mathbf{c l}(W(\mathbf{A}))$, there exists a unit vector $\mathbf{x}_{1} \in \mathcal{H}$ such that $\left\|\left\langle\mathbf{A x}_{1}, \mathbf{x}_{1}\right\rangle\right\|<1 / 2$. Suppose we have an orthonormal set $\left\{\mathbf{x}_{1}, \ldots, \mathbf{x}_{n}\right\}$ such that $\left\|\left\langle\mathbf{A x}_{n}, \mathbf{x}_{n}\right\rangle\right\|<1 / 2^{n}$. Let $Q$ be the orthogonal projection of $\mathcal{H}$ onto the subspace $S$ spanned by $\mathbf{x}_{1}, \ldots, \mathbf{x}_{n}$ and let

$$
\mathbf{B}=\left(\left.(I-Q) A_{1}(I-Q)\right|_{S^{\perp}}, \ldots,\left.(I-Q) A_{m}(I-Q)\right|_{S^{\perp}}\right) .
$$

Let $\mathbf{b}=\left(b_{1}, \ldots, b_{m}\right) \in W_{3}(\mathbf{B})$ and $\mathbf{b} I_{S}=\left(b_{1} I_{S}, \ldots, b_{m} I_{S}\right)$. Then for $\bar{Q}=$ $I-Q$, we have

$$
\mathbf{b} I_{S} \oplus \mathbf{B}=\left(b_{1} Q+\bar{Q} A_{1} \bar{Q}, \ldots, b_{m} Q+\bar{Q} A_{m} \bar{Q}\right)=\mathbf{A}+\mathbf{F}
$$

for some $\mathbf{F} \in \mathcal{F}(\mathcal{H})^{m} \cap \mathcal{S}(\mathcal{H})^{m}$. Therefore, $\mathbf{0} \in \mathbf{c l}\left(W\left(\mathbf{b} I_{S} \oplus \mathbf{B}\right)\right)$. Hence, there exists a unit vector $\mathbf{x} \in \mathcal{H}$ such that $\|\langle(\mathbf{A}+\mathbf{F}) \mathbf{x}, \mathbf{x}\rangle\|<1 / 2^{n+2}$. Let $\mathbf{x}=\mathbf{y}+\mathbf{z}$, where $\mathbf{y} \in S$ and $\mathbf{z} \in S^{\perp}$. Then $\|\mathbf{y}\|^{2}+\|\mathbf{z}\|^{2}=\|\mathbf{x}\|^{2}=1$. If $\mathbf{z}=\mathbf{0}$, then $\langle(\mathbf{A}+\mathbf{F}) \mathbf{x}, \mathbf{x}\rangle=\mathbf{b} \in W_{3}(\mathbf{B}) \subseteq \mathbf{c l}(W(\mathbf{B}))$. If $\mathbf{z} \neq \mathbf{0}$, then by Theorem 2.2 , we have

$$
\langle(\mathbf{A}+\mathbf{F}) \mathbf{x}, \mathbf{x}\rangle=\|\mathbf{y}\|^{2} \mathbf{b}+\|\mathbf{z}\|^{2}\langle\mathbf{B}(\mathbf{z} /\|\mathbf{z}\|), \mathbf{z} /\|\mathbf{z}\|\rangle \in \mathbf{c l}(W(\mathbf{B})) .
$$

So there exists a unit vector $\mathbf{x}_{n+1} \in S^{\perp}$ such that

$$
\left\|\langle(\mathbf{A}+\mathbf{F}) \mathbf{x}, \mathbf{x}\rangle-\left\langle\mathbf{B} \mathbf{x}_{n+1}, \mathbf{x}_{n+1}\right\rangle\right\|<\frac{1}{2^{n+2}},
$$

and hence

$$
\left\|\left\langle\mathbf{A} \mathbf{x}_{n+1}, \mathbf{x}_{n+1}\right\rangle\right\|=\left\|\left\langle\mathbf{B} \mathbf{x}_{n+1}, \mathbf{x}_{n+1}\right\rangle\right\|<\frac{1}{2^{n+1}},
$$

because $\left\langle\mathbf{F} \mathbf{x}_{n+1}, \mathbf{x}_{n+1}\right\rangle=\mathbf{0}$. Inductively, we can choose an orthonormal sequence $\left\{\mathbf{x}_{n}\right\}_{n=1}^{\infty}$ such that

$$
\left\|\left\langle\mathbf{A} \mathbf{x}_{n}, \mathbf{x}_{n}\right\rangle\right\|<\frac{1}{2^{n}} \quad \text { for all } n \geq 1 .
$$

Let $n_{1}=1$. For every $1 \leq i \leq m$, we have

$$
\sum_{n=1}^{\infty}\left|\left\langle A_{i} \mathbf{x}_{n_{1}}, \mathbf{x}_{n}\right\rangle\right|^{2} \leq\left\|A_{i} \mathbf{x}_{n_{1}}\right\|^{2} \text { and } \sum_{n=1}^{\infty}\left|\left\langle A_{i} \mathbf{x}_{n}, \mathbf{x}_{n_{1}}\right\rangle\right|^{2} \leq\left\|A_{i}^{*} \mathbf{x}_{n_{1}}\right\|^{2} .
$$

Hence, there exists $n_{2}>n_{1}$ such that

$$
\sum_{n=n_{2}}^{\infty}\left|\left\langle A_{i} \mathbf{x}_{n_{1}}, \mathbf{x}_{n}\right\rangle\right|^{2}<\frac{1}{2} \text { and } \sum_{n=n_{2}}^{\infty}\left|\left\langle A_{i} \mathbf{x}_{n}, \mathbf{x}_{n_{1}}\right\rangle\right|^{2}<\frac{1}{2}
$$

for all $1 \leq i \leq m$. Repeating this procedure, we get a strictly increasing sequence $\left\{n_{k}\right\}_{k=1}^{\infty}$ of positive integers such that for all $1 \leq i \leq m$, we have

$$
\sum_{n=n_{k+1}}^{\infty}\left|\left\langle A_{i} \mathbf{x}_{n_{k}}, \mathbf{x}_{n}\right\rangle\right|^{2}<\frac{1}{2^{k}} \text { and } \sum_{n=n_{k+1}}^{\infty}\left|\left\langle A_{i} \mathbf{x}_{n}, \mathbf{x}_{n_{k}}\right\rangle\right|^{2}<\frac{1}{2^{k}} .
$$


Formulas (1) and (2) imply that

$$
\sum_{k, l=1}^{\infty}\left|\left\langle A_{i} \mathbf{x}_{n_{k}}, \mathbf{x}_{n_{l}}\right\rangle\right|^{2}<\infty .
$$

Let $P$ be the orthogonal projection onto the subspace spanned by $\left\{\mathbf{x}_{n_{k}}\right\}_{k=1}^{\infty}$. Then it follows from (3) that $P A_{i} P$ is compact for all $1 \leq i \leq m$.

\section{Convexity and star-shapedness}

Theorem 3.1. Let $\mathbf{A} \in \mathcal{S}(\mathcal{H})^{m}$. Then $W_{\mathrm{e}}(\mathbf{A})$ is a compact convex subset of $\mathbf{c l}(W(\mathbf{A}))$. Moreover, each element in $W_{\mathrm{e}}(\mathbf{A})$ is a star center of the starshaped set $\mathbf{c l}(W(\mathbf{A}))$.

Proof. Because $W_{\mathrm{e}}(\mathbf{A})$ is the intersection of compact sets, it is compact. To prove the convexity, let $\mathbf{a}, \mathbf{b} \in W_{\mathrm{e}}(\mathbf{A})$ and $0 \leq t \leq 1$. Then for every $\mathbf{F} \in \mathcal{F}(\mathcal{H})^{m} \cap \mathcal{S}(\mathcal{H})^{m}$, we have $\mathbf{a} \in W_{\mathrm{e}}(\mathbf{A})=W_{\mathrm{e}}(\mathbf{A}+\mathbf{F})$ and $\mathbf{b} \in W_{\mathrm{e}}(\mathbf{A}) \subseteq$ $\operatorname{cl}(W(\mathbf{A}+\mathbf{F}))$. So, by Theorem 2.2 , we have $t \mathbf{a}+(1-t) \mathbf{b} \in \mathbf{c l}(W(\mathbf{A}+\mathbf{F}))$. Hence,

$$
t \mathbf{a}+(1-t) \mathbf{b} \in \bigcap\left\{\mathbf{c l}(W(\mathbf{A}+\mathbf{F})): \mathbf{F} \in \mathcal{F}(\mathcal{H})^{m} \cap \mathcal{S}(\mathcal{H})^{m}\right\}=W_{\mathrm{e}}(\mathbf{A}) .
$$

By Theorems 2.1 and 2.2, we have the last assertion.

Note that $W_{\mathrm{e}}(\mathbf{A}) \cap W(\mathbf{A})$ may be empty. For example, if

$$
A=\operatorname{diag}(1,1 / 2,1 / 3, \ldots)
$$

acts on $\ell^{2}$, then $W_{\mathrm{e}}(A)=\{0\}$ and $W(A)=(0,1]$. One may wonder whether a point $\mathbf{a} \in W_{\mathrm{e}}(\mathbf{A}) \cap W(\mathbf{A})$ is a star center of $W(\mathbf{A})$. This is not true, as shown by the example below. Moreover, the example shows that for $m \geq 4$ there exists $\mathbf{A} \in \mathcal{S}(\mathcal{H})^{m}$ such that $\operatorname{cl}(W(\mathbf{A}))$ is convex whereas $W(\mathbf{A})$ is not. Of course, this is impossible for $m \leq 3$ as $W(\mathbf{A})$ is always convex.

Example 3.2. Consider $\mathcal{H}=\ell^{2}$ with canonical basis $\left\{e_{n}: n \geq 1\right\}$. Let $\mathbf{A}=\left(A_{1}, \ldots, A_{4}\right)$ with

$$
\begin{array}{ll}
A_{1}=\operatorname{diag}(1,0,1 / 3,1 / 4, \ldots), & A_{2}=\operatorname{diag}(1,0) \oplus \mathbf{0}, \\
A_{3}=\left(\begin{array}{ll}
0 & 1 \\
1 & 0
\end{array}\right) \oplus \mathbf{0}, & A_{4}=\left(\begin{array}{cc}
0 & i \\
-i & 0
\end{array}\right) \oplus \mathbf{0} .
\end{array}
$$

Then $(1,1,0,0) \in W(\mathbf{A})$ and $(0,0,0,0) \in W(\mathbf{A}) \cap W_{\mathrm{e}}(\mathbf{A})$, but $(1 / 2,1 / 2,0,0)$ $\notin W(\mathbf{A})$. Hence, $W(\mathbf{A})$ is not convex. However, $\mathbf{c l}(W(\mathbf{A}))$ is convex.

Proof. Note that $(1,1,0,0)=\left\langle\mathbf{A} e_{1}, e_{1}\right\rangle \in W(\mathbf{A})$ and

$$
(0,0,0,0)=\left\langle\mathbf{A} e_{2}, e_{2}\right\rangle=\lim _{n \rightarrow \infty}\left\langle\mathbf{A} e_{n}, e_{n}\right\rangle \in W(\mathbf{A}) \cap W_{\mathrm{e}}(\mathbf{A}) .
$$


To show that $(1 / 2,1 / 2,0,0) \notin W(\mathbf{A})$, consider a unit vector $\mathbf{x}=\sum x_{j} e_{j}$ such that $\sum_{n=1}^{\infty}\left|x_{n}\right|^{2}=1$. If $\left\langle A_{1} \mathbf{x}, \mathbf{x}\right\rangle=\left\langle A_{2} \mathbf{x}, \mathbf{x}\right\rangle=1 / 2$, then

$$
\left|x_{1}\right|^{2}+\sum_{n=3}^{\infty}\left|x_{n}\right|^{2} / n=\left|x_{1}\right|^{2}=1 / 2 .
$$

Thus, $x_{n}=0$ for all $n \geq 3$ and $\left|x_{1}\right|^{2}=\left|x_{2}\right|^{2}=1 / 2$. It then follows that $\left(\left\langle A_{3} \mathbf{x}, \mathbf{x}\right\rangle,\left\langle A_{4} \mathbf{x}, \mathbf{x}\right\rangle\right) \neq(0,0)$. This proves that $(1 / 2,1 / 2,0,0) \notin W(\mathbf{A})$. Hence, $(0,0,0,0) \in W_{\mathrm{e}}(\mathbf{A}) \cap W(\mathbf{A})$ is not a star center of $W(\mathbf{A})$, and $W(\mathbf{A})$ is not convex.

To see that $\mathbf{c l}(W(\mathbf{A}))$ is convex, note that $\mathbf{0} \in W_{\mathrm{e}}(\mathbf{A})$. Thus, by Theorem 3.1 , for every $\mathbf{b} \in \mathbf{c l}(W(\mathbf{A}))$ we have $t \mathbf{0}+(1-t) \mathbf{b} \in \mathbf{c l}(W(\mathbf{A}))$ for any $t \in[0,1]$.

Let $\mathbf{B}=\left(B_{1}, B_{2}, B_{3}, B_{4}\right)$, where

$$
\begin{array}{ll}
B_{1}=\operatorname{diag}(0,1,0), & B_{2}=\operatorname{diag}(0,1,0), \\
B_{3}=[0] \oplus\left(\begin{array}{ll}
0 & 1 \\
1 & 0
\end{array}\right), & B_{4}=[0] \oplus\left(\begin{array}{cc}
0 & i \\
-i & 0
\end{array}\right),
\end{array}
$$

and $\mathbf{C}=\left(C_{1}, C_{2}, C_{3}, C_{4}\right)$, where $C_{1}=\operatorname{diag}(1 / 3,1 / 4, \ldots) \oplus[0], C_{2}=C_{3}=$ $C_{4}=\operatorname{diag}(0,0, \ldots) \oplus[0]$. Then it is easy to verify that

$$
W(\mathbf{B})=\left\{(r, r, s, t) \in \mathbb{R}^{4}: 4(r-1 / 2)^{2}+s^{2}+t^{2} \leq 1\right\}
$$

and

$$
W(\mathbf{C})=\{(c, 0,0,0): c \in[0,1 / 3]\}
$$

are both compact and convex. Hence, $W(\mathbf{B} \oplus \mathbf{C})=\operatorname{conv}(W(\mathbf{B}) \cup W(\mathbf{C}))$ is compact and convex and

$$
W(\mathbf{A}) \subseteq W(\mathbf{B} \oplus \mathbf{C}) \Rightarrow \mathbf{c l}(W(\mathbf{A})) \subseteq W(\mathbf{B} \oplus \mathbf{C}) .
$$

On the other hand, $\mathbf{B} \oplus \mathbf{C}=[0] \oplus \mathbf{A} \oplus[0]$. Therefore,

$$
W(\mathbf{B} \oplus \mathbf{C})=\{t \mathbf{0}+(1-t) \mathbf{b}: \mathbf{b} \in W(\mathbf{A})\} \subseteq \mathbf{c l}(W(\mathbf{A})) .
$$

So, $\operatorname{cl}(W(\mathbf{A}))=W(\mathbf{B} \oplus \mathbf{C})$ is convex.

4. Other descriptions of $W_{\mathrm{e}}(\mathbf{A})$. For $\mathbf{c}=\left(c_{1}, \ldots, c_{m}\right) \in \mathbb{R}^{m}$ and $\mathbf{A}=$ $\left(A_{1}, \ldots, A_{m}\right) \in \mathcal{S}(\mathcal{H})^{m}$, let $\mathbf{c} \cdot \mathbf{A}=\sum_{i=1}^{m} c_{i} A_{i}$. Using the convexity of $W_{\mathrm{e}}(\mathbf{A})$, we obtain additional conditions equivalent to membership in $W_{\mathrm{e}}(\mathbf{A})$ in terms of $\mathbf{c} \cdot \mathbf{A} \in \mathcal{S}(\mathcal{H})$ so that the joint behavior of $A_{1}, \ldots, A_{m}$ can be understood from their linear combinations. For $A \in \mathcal{S}(\mathcal{H})$ and a positive integer $k$, let

$$
\lambda_{k}(A)=\inf \{\max \sigma(A+F): F \in \mathcal{S}(\mathcal{H}) \text { with } \operatorname{rank}(F)<k\} .
$$

Theorem 4.1. Let $\mathbf{A} \in \mathcal{S}(\mathcal{H})^{m}$ and $\mathbf{a}=\left(a_{1}, \ldots, a_{m}\right) \in \mathbb{R}^{m}$. Then $\mathbf{a} \in W_{\mathrm{e}}(\mathbf{A})$ if and only if any one (and hence all) of the following conditions holds: 
(1) For every $\mathbf{c} \in \mathbb{R}^{m}, \mathbf{c} \cdot \mathbf{a} \in W_{\mathrm{e}}(\mathbf{c} \cdot \mathbf{A})$.

(2) For every $\mathbf{c} \in \mathbb{R}^{m}, \mathbf{c} \cdot \mathbf{a} \in \bigcap\{\mathbf{c l}(W(\mathbf{c} \cdot \mathbf{A}+F)): F \in \mathcal{F}(\mathcal{H}) \cap \mathcal{S}(\mathcal{H})\}$.

(3) For every $\mathbf{c} \in \mathbb{R}^{m}$, there is an orthonormal sequence $\left\{\mathbf{x}_{n}\right\}_{n=1}^{\infty} \subset \mathcal{H}$ such that

$$
\lim _{n \rightarrow \infty}\left\langle\mathbf{c} \cdot \mathbf{A} \mathbf{x}_{n}, \mathbf{x}_{n}\right\rangle=\mathbf{c} \cdot \mathbf{a} .
$$

(4) For every $\mathbf{c} \in \mathbb{R}^{m}$, there is a sequence $\left\{\mathbf{x}_{n}\right\}_{n=1}^{\infty} \subset \mathcal{H}$ of unit vectors such that $\left\{\mathbf{x}_{n}\right\}_{n=1}^{\infty}$ converges weakly to $\mathbf{0}$ in $\mathcal{H}$ and

$$
\lim _{n \rightarrow \infty}\left\langle\mathbf{c} \cdot \mathbf{A} \mathbf{x}_{n}, \mathbf{x}_{n}\right\rangle=\mathbf{c} \cdot \mathbf{a} .
$$

(5) For every $\mathbf{c} \in \mathbb{R}^{m}$, there is an infinite-dimensional projection $P \in$ $\mathcal{S}(\mathcal{H})$ such that $P(\mathbf{c} \cdot \mathbf{A}-\mathbf{c} \cdot \mathbf{a} I) P \in \mathcal{K}(\mathcal{H})$.

(6) For every $\mathbf{c} \in \mathbb{R}^{m}$ and $k \geq 1, \lambda_{k}(\mathbf{c} \cdot \mathbf{A}-\mathbf{c} \cdot \mathbf{a} I) \geq 0$.

Proof. By the convexity of $W_{\mathrm{e}}(\mathbf{A})$, we can apply the separation theorem to Theorem 2.1 to show that $\mathbf{a} \in W_{\mathrm{e}}(\mathbf{A})$ if and only if any one of the conditions (1) to (5) holds.

To prove the equivalence of condition (6), suppose $\mathbf{a} \in \mathbb{R}^{m}$. Without loss of generality, we may assume that $\mathbf{a}=\mathbf{0}$. Suppose $\mathbf{0}$ satisfies condition (6). Then for every $\mathbf{c} \in \mathbb{R}^{m}$ and $F \in \mathcal{F}(\mathcal{H}) \cap \mathcal{S}(\mathcal{H})$ with $\operatorname{rank}(F)=k$, we have $\lambda_{1}(\mathbf{c} \cdot \mathbf{A}+F) \geq \lambda_{k+1}(\mathbf{c} \cdot \mathbf{A}) \geq 0 \quad$ and $\quad \lambda_{1}(-(\mathbf{c} \cdot \mathbf{A}+F)) \geq \lambda_{k+1}(-\mathbf{c} \cdot \mathbf{A}) \geq 0$. Hence, $\mathbf{c} \cdot \mathbf{0}=0 \in \mathbf{c l}(W(\mathbf{c} \cdot \mathbf{A}+F))$. Therefore, condition (2) is satisfied.

Conversely, if $\mathbf{0}$ does not satisfy condition (6), then there exist $\mathbf{c} \in \mathbb{R}^{m}$ and $k \geq 1$ such that $\lambda_{k}(\mathbf{c} \cdot \mathbf{A})<0$. Thus there exists $F \in \mathcal{F}(\mathcal{H}) \cap \mathcal{S}(\mathcal{H})$ such that $\mathbf{c} \cdot \mathbf{A}+F<0$ and $\mathbf{0}$ does not satisfy condition (2).

Let $\mathbf{A} \in \mathcal{S}(\mathcal{H})^{m}$. Although the set $\mathbf{c l}(W(\mathbf{A}))$ may not be convex if $m \geq 4$, we have the following analog of the separation theorem for a convex set.

Theorem 4.2. Let $\mathbf{A}=\left(A_{1}, \ldots, A_{m}\right) \in \mathcal{S}(\mathcal{H})^{m}$ and $\mathbf{d}=\left(d_{1}, \ldots, d_{m}\right)$ $\in \mathbb{R}^{m}$. Then $\mathbf{d} \notin W_{\mathrm{e}}(\mathbf{A})$ if and only if any one (and hence all) of the following conditions holds:

(a) There exists $\mathbf{K} \in \mathcal{K}(\mathcal{H})^{m} \cap \mathcal{S}(\mathcal{H})^{m}$ such that $\mathbf{d} \notin \mathbf{c l}(W(\mathbf{A}+\mathbf{K}))$.

(b) There exists $\mathbf{F} \in \mathcal{F}(\mathcal{H})^{m} \cap \mathcal{S}(\mathcal{H})^{m}$ with $\mathbf{d} \notin \mathbf{c o n v}(\mathbf{c l}(W(\mathbf{A}+\mathbf{F})))$.

(c) There exist $F \in \mathcal{F}(\mathcal{H}) \cap \mathcal{S}(\mathcal{H}), r>0$ and $\mathbf{c}=\left(c_{1}, \ldots, c_{m}\right) \in \mathbb{R}^{m}$ such that

$$
\left(\sum_{i=1}^{m} c_{i}\left(A_{i}-d_{i} I\right)\right)+F>r I_{\mathcal{H}} .
$$

Proof. For simplicity, replace $\left(A_{1}, \ldots, A_{m}\right)$ by $\left(A_{1}-d_{1} I, \ldots, A_{m}-d_{m} I\right)$ and assume that $\mathbf{d}=(0, \ldots, 0)$. 
(c) $\Rightarrow$ (b). If (c) holds, we may perturb $\left(c_{1}, \ldots, c_{m}\right)$ so that $c_{j} \neq 0$ for all $j \in\{1, \ldots, m\}$ and condition (4) still holds true. In particular, $c_{1} \neq 0$. Then let $\mathbf{F}=\left(F / c_{1}, 0, \ldots, 0\right)$. We have $\mathbf{c} \cdot \mathbf{a}>r>0$ for all $\mathbf{a} \in W(\mathbf{A}+\mathbf{F})$. Therefore, $\mathbf{0} \notin \operatorname{conv}(\mathbf{c l}(W(\mathbf{A}+\mathbf{F})))$.

Clearly, we have $(\mathrm{b}) \Rightarrow(\mathrm{a})$, which implies that $\mathbf{0} \notin W_{\mathrm{e}}(\mathbf{A})$.

Finally, suppose $\mathbf{0} \notin W_{\mathrm{e}}(\mathbf{A})$. Then by Theorem $4.1(2)$, there exist a real vector $\mathbf{c}=\left(c_{1}, \ldots, c_{m}\right)$ and $F \in \mathcal{F}(\mathcal{H}) \cap \mathcal{S}(\mathcal{H})$ such that $0=\mathbf{c} \cdot \mathbf{0} \notin$ $\operatorname{cl}(W(\mathbf{c} \cdot \mathbf{A}+F))$. Since $\mathbf{c l}(W(\mathbf{c} \cdot \mathbf{A}+F))$ is a closed subinterval $[s, t]$ of $\mathbb{R}$, we may assume that $0<s \leq t$. Let $r=s / 2$. Then $\left(\sum_{i=1}^{m} c_{i} A_{i}\right)+F>r I_{\mathcal{H}}$. Hence, (c) holds.

Let $\Omega=\left\{\mathbf{c} \in \mathbb{R}^{m}:\langle\mathbf{c}, \mathbf{c}\rangle=1\right\}$. By Theorem 4.2, we have the following result showing that $W_{\mathrm{e}}(\mathbf{A})$ can be expressed as the intersection of halfspaces.

Corollary 4.3. Let $\mathbf{A}=\left(A_{1}, \ldots, A_{m}\right) \in \mathcal{S}(\mathcal{H})^{m}$. Then

$$
\begin{aligned}
W_{\mathrm{e}}(\mathbf{A}) & =\bigcap_{\mathbf{c} \in \Omega}\left\{\mathbf{d} \in \mathbb{R}^{m}:\langle\mathbf{c}, \mathbf{d}\rangle \leq \max W_{\mathrm{e}}(\mathbf{c} \cdot \mathbf{A})\right\} \\
& =\left\{\mathbf{d} \in \mathbb{R}^{m}:\langle\mathbf{c}, \mathbf{d}\rangle \in W_{\mathrm{e}}(\mathbf{c} \cdot \mathbf{A}) \text { for all } \mathbf{c} \in \Omega\right\} .
\end{aligned}
$$

For $A \in \mathcal{B}(\mathcal{H})$, let $\sigma_{\mathrm{e}}(A)=\bigcap\{\sigma(A+K): K \in \mathcal{K}(\mathcal{H})\}$ be the essential spectrum of $A$. Then for $A \in \mathcal{S}(\mathcal{H})$, we have

$$
W_{\mathrm{e}}(A)=\operatorname{conv} \sigma_{\mathrm{e}}(A) .
$$

Thus, one may replace $\max W_{\mathrm{e}}(\mathbf{c} \cdot \mathbf{A})$ by $\max \sigma_{\mathrm{e}}(\mathbf{c} \cdot \mathbf{A})$ in Corollary 4.3.

Corollary 4.4. Let $\mathbf{A}=\left(A_{1}, \ldots, A_{m}\right) \in \mathcal{S}(\mathcal{H})^{m}$. If $\mathbf{d} \notin \mathbf{c l}(W(\mathbf{A}))$, then for any $i \in\{1, \ldots, m\}$ there exists $F \in \mathcal{F}(\mathcal{H}) \cap \mathcal{S}(\mathcal{H})$ such that $\mathbf{d} \notin$ $\operatorname{conv}(\mathbf{c l}(W(\tilde{\mathbf{A}})))$, where $\tilde{\mathbf{A}}=\left(A_{1}, \ldots, A_{i-1}, A_{i}+F, A_{i+1}, \ldots, A_{m}\right)$.

Proof. If $\mathbf{d} \notin \mathbf{c l}(W(\mathbf{A}))$, then $\mathbf{d} \notin W_{\mathrm{e}}(\mathbf{A})$. The result readily follows from the arguments in the last paragraph in the proof of Theorem 4.2.

It follows from Theorem 2.1 that the intersection of the non-convex sets $\operatorname{cl}(W(\mathbf{A}+\mathbf{K}))$, which equals $W_{\mathrm{e}}(\mathbf{A})$, is a convex set. By Theorem 4.2 and Corollary 4.4, we see that one can replace $\mathbf{c l}(W(\mathbf{A}+\mathbf{K}))$ by its convex hull in the intersection to obtain the same convex set $W_{\mathrm{e}}(\mathbf{A})$. It is known that for any $\mathbf{B}=\left(B_{1}, \ldots, B_{m}\right) \in \mathcal{B}(\mathcal{H})^{m}$,

$$
\operatorname{conv}(\mathbf{c l}(W(\mathbf{B})))=\left\{\left(f\left(B_{1}\right), \ldots, f\left(B_{m}\right)\right): f \in \Xi\right\},
$$

where $\Xi$ is the set of linear functionals $f$ on $\mathcal{B}(\mathcal{H})$ satisfying $1=f(I)=$ $\max \{f(X): X \in \mathcal{B}(\mathcal{H}),\|X\| \leq 1\}$ (for example, see [10,11]). So, it is easier to determine $\mathbf{c o n v}(\mathbf{c l}(W(\mathbf{A}+\mathbf{K})))$ than $\operatorname{cl}(W(\mathbf{A}+\mathbf{K}))$. In fact, we have the following. 
Corollary 4.5. Let $\mathbf{A} \in \mathcal{S}(\mathcal{H})^{m}$ and $i \in\{1, \ldots, m\}$. Then

$$
\begin{aligned}
W_{\mathrm{e}}(\mathbf{A}) & =\bigcap\left\{\mathbf{c l}(W(\mathbf{A}+\mathbf{F})): \mathbf{F} \in\{0\}^{i-1} \times(\mathcal{F}(\mathcal{H}) \cap \mathcal{S}(\mathcal{H})) \times\{0\}^{m-i}\right\} \\
= & \bigcap\left\{\mathbf{c o n v}(\mathbf{c l}(W(\mathbf{A}+\mathbf{F}))): \mathbf{F} \in\{0\}^{i-1} \times(\mathcal{F}(\mathcal{H}) \cap \mathcal{S}(\mathcal{H})) \times\{0\}^{m-i}\right\} .
\end{aligned}
$$

Proof. Let $\mathbf{F} \in\{0\}^{i-1} \times(\mathcal{F}(\mathcal{H}) \cap \mathcal{S}(\mathcal{H})) \times\{0\}^{m-i}$. Clearly,

$$
W_{\mathrm{e}}(\mathbf{A}) \subseteq \mathbf{c l}(W(\mathbf{A}+\mathbf{F})) \subseteq \mathbf{c o n v}(\mathbf{c l}(W(\mathbf{A}+\mathbf{F}))) .
$$

So, we may take the intersection of the second and third sets over all $\mathbf{F} \in$ $\{0\}^{i-1} \times(\mathcal{F}(\mathcal{H}) \cap \mathcal{S}(\mathcal{H})) \times\{0\}^{m-i}$, and get an inclusion involving the three sets in the corollary. Finally, if $\mathbf{d} \notin W_{\mathrm{e}}(\mathbf{A})$, then $\mathbf{d}$ will not belong to the third set by Corollary 4.4. So, the third set is a subset of $W_{\mathrm{e}}(\mathbf{A})$. Hence, the three sets in the corollary are equal.

5. Additional results. The following result shows that $W_{\mathrm{e}}(\mathbf{A})$ is unchanged under certain operations on $\mathbf{A}$.

Theorem 5.1. Let $\mathbf{A}=\left(A_{1}, \ldots, A_{m}\right) \in \mathcal{S}(\mathcal{H})^{m}$.

(a) Suppose $\mathcal{H}_{1}$ is a closed subspace of $\mathcal{H}$ such that $\mathcal{H}_{1}^{\perp}$ is finite-dimensional. If $X: \mathcal{H}_{1} \rightarrow \mathcal{H}$ is such that $X^{*} X=I_{\mathcal{H}_{1}}$, then

$$
W_{\mathrm{e}}(\mathbf{A})=W_{\mathrm{e}}\left(X^{*} A_{1} X, \ldots, X^{*} A_{m} X\right) .
$$

(b) For each $j \in\{1, \ldots, m\}$, suppose $P_{j}: \mathcal{H} \rightarrow \mathcal{H}$ is an orthogonal projection such that $I-P_{j}$ has finite rank. Then

$$
W_{\mathrm{e}}(\mathbf{A})=W_{\mathrm{e}}\left(P_{1} A_{1} P_{1}, \ldots, P_{m} A_{m} P_{m}\right) .
$$

Proof. Use Theorem 2.1.

We will establish some additional relationships between the sets $W_{\mathrm{e}}(\mathbf{A})$ and $W(\mathbf{A})$. The next theorem generalizes the results of [29] and [14].

Theorem 5.2. Let $\mathbf{A} \in \mathcal{S}(\mathcal{H})^{m}$. Then $W_{\mathrm{e}}(\mathbf{A})=\operatorname{cl}(W(\mathbf{A}))$ if and only if $\operatorname{Ext}(W(\mathbf{A})) \subseteq W_{\mathrm{e}}(\mathbf{A})$.

Proof. If $W_{\mathrm{e}}(\mathbf{A})=\mathbf{c l}(W(\mathbf{A}))$, then

$$
\operatorname{Ext}(W(\mathbf{A})) \subseteq W(\mathbf{A}) \subseteq W_{\mathrm{e}}(\mathbf{A}) .
$$

Conversely, if $\operatorname{Ext}(W(\mathbf{A})) \subseteq W_{\mathrm{e}}(\mathbf{A})$, then by $(\mathrm{P} 5)$,

$$
\operatorname{Ext}(\mathbf{c l}(W(\mathbf{A}))) \subseteq W_{\mathrm{e}}(\mathbf{A}) .
$$

Hence,

$$
\operatorname{cl}(W(\mathbf{A})) \subseteq \operatorname{conv}\left(\operatorname{Ext}(\mathbf{c l}(W(\mathbf{A}))) \subseteq \operatorname{conv}\left(W_{\mathrm{e}}(\mathbf{A})\right)=W_{\mathrm{e}}(\mathbf{A}) .\right.
$$

Since $W_{\mathrm{e}}(\mathbf{A}) \subseteq \operatorname{cl}(W(\mathbf{A}))$, we have $W_{\mathrm{e}}(\mathbf{A})=\operatorname{cl}(W(\mathbf{A}))$. 
For $k \geq 1$, let $I_{k}$ denote the $k \times k$ identity matrix. Then for $\mathbf{A}=$ $\left(A_{1}, \ldots, A_{m}\right) \in \mathcal{S}(\mathcal{H})^{m}$, we have

$$
\mathbf{A} \otimes I_{k}=\left(A_{1} \otimes I_{k}, \ldots, A_{m} \otimes I_{k}\right) \in \mathcal{S}(\underbrace{\mathcal{H} \oplus \cdots \oplus \mathcal{H}}_{k})^{m} .
$$

Similarly, let $I_{\infty}$ denote the identity operator acting on $\ell_{2}$. Then for $\mathbf{A}=$ $\left(A_{1}, \ldots, A_{m}\right) \in \mathcal{S}(\mathcal{H})^{m}$, we have

$$
\mathbf{A} \otimes I_{\infty}=\left(A_{1} \otimes I_{\infty}, \ldots, A_{m} \otimes I_{\infty}\right) \in \mathcal{S}(\underbrace{\mathcal{H} \oplus \mathcal{H} \oplus \cdots}_{\text {infinitely many }})^{m} .
$$

Theorem 5.3. Let $\mathbf{A}=\left(A_{1}, \ldots, A_{m}\right) \in \mathcal{S}(\mathcal{H})^{m}$. Then for any positive integer $k>\sqrt{m}-1$,

$$
W\left(\mathbf{A} \otimes I_{k}\right)=\operatorname{conv}(W(\mathbf{A}))
$$

Moreover,

$$
W_{\mathrm{e}}\left(\mathbf{A} \otimes I_{\infty}\right)=\operatorname{cl}(\operatorname{conv}(W(\mathbf{A}))) .
$$

Proof. Suppose $k>\sqrt{m}-1$. By the result in [34], every $\mathbf{a} \in \operatorname{conv}(W(\mathbf{A}))$ can be written as $\mathbf{a}=\sum_{j=1}^{k} t_{j}\left\langle\mathbf{A} \mathbf{x}_{j}, \mathbf{x}_{j}\right\rangle$ for some unit vectors $\mathbf{x}_{1}, \ldots, \mathbf{x}_{k}$ $\in \mathcal{H}$. Thus, for $\mathbf{x}=\left(\sqrt{t_{1}} \mathbf{x}_{1}, \ldots, \sqrt{t_{k}} \mathbf{x}_{k}\right) \in \mathcal{H} \oplus \cdots \oplus \mathcal{H}$, we have $\langle\mathbf{A} \otimes$ $\left.I_{k} \mathbf{x}, \mathbf{x}\right\rangle=\mathbf{a}$. Conversely, if $\mathbf{a}=\left\langle\mathbf{A} \otimes I_{k} \mathbf{x}, \mathbf{x}\right\rangle \in W\left(\mathbf{A} \otimes I_{k}\right)$, one can decompose the unit vector $\mathbf{x}$ into $k$ parts $\mathbf{y}_{1}, \ldots, \mathbf{y}_{k}$ according to the structure of $\mathcal{H} \otimes I_{k}$. Then

$$
\mathbf{a}=\sum_{j=1}^{k}\left\|\mathbf{y}_{j}\right\|^{2}\left\langle A \mathbf{y}_{j} /\left\|\mathbf{y}_{j}\right\|, \mathbf{y}_{j} /\left\|\mathbf{y}_{j}\right\|\right\rangle \in \operatorname{conv}(W(\mathbf{A}))
$$

If $\mathbf{a} \in \operatorname{cl}(\operatorname{conv}(W(\mathbf{A})))$, then there is a sequence $\left\{\mathbf{x}_{n}\right\}$ of unit vectors in $\mathcal{H}$ such that $\left\langle\mathbf{A} \mathbf{x}_{n}, \mathbf{x}_{n}\right\rangle \rightarrow \mathbf{a}$. Let

$$
\tilde{\mathbf{x}}_{n}=(\underbrace{0, \ldots, 0}_{n-1}, \mathbf{x}_{n}, 0, \ldots) \in \mathcal{H} \oplus \mathcal{H} \oplus \cdots .
$$

Then $\left\{\tilde{\mathbf{x}}_{n}\right\}$ is an orthonormal sequence in $\mathcal{H} \oplus \mathcal{H} \oplus \cdots$ and $\left\langle\mathbf{A} \otimes I_{\infty} \tilde{\mathbf{x}}_{n}, \tilde{\mathbf{x}}_{n}\right\rangle$ $\rightarrow \mathbf{a}$. Therefore, $\mathbf{a} \in W_{\mathrm{e}}\left(\mathbf{A} \otimes I_{\infty}\right)$. Since

$W_{\mathrm{e}}\left(\mathbf{A} \otimes I_{\infty}\right) \subseteq \mathbf{c l}\left(W\left(\mathbf{A} \otimes I_{\infty}\right)\right)=\mathbf{c l}\left(\bigcup_{k=1}^{\infty} W\left(\mathbf{A} \otimes I_{k}\right)\right) \subseteq \mathbf{c l}(\mathbf{c o n v}(W(\mathbf{A})))$, we get the reverse inclusion.

COROLlary 5.4. Let $S$ be a compact convex subset of $\mathbb{R}^{m}$. Then there are $\mathbf{A}, \tilde{\mathbf{A}} \in \mathcal{S}(\mathcal{H})^{m}$ with $\mathcal{H}=\ell^{2}$ such that $W(\mathbf{A})$ is convex and

$$
W(\mathbf{A}) \subseteq S=\operatorname{cl}(W(\mathbf{A}))=W_{\mathrm{e}}(\tilde{\mathbf{A}}) .
$$

Proof. For $j=1, \ldots, m$, let $A_{j}=\operatorname{diag}\left(a_{1 j}, a_{2 j}, \ldots\right)$ act on $\ell^{2}$ with the standard canonical basis $\left\{e_{n}: n \geq 1\right\}$ and be such that $\left\{\left(a_{i 1}, \ldots, a_{i m}\right)\right.$ : 
$i \geq 1\}$ is a dense subset of $S$. Then for $\mathbf{A}=\left(A_{1}, \ldots, A_{m}\right)$ the set

$$
W(\mathbf{A})=\operatorname{conv}\left\{\left(a_{i 1}, \ldots, a_{i m}\right): i \geq 1\right\}
$$

is convex, and $\tilde{\mathbf{A}}=\mathbf{A} \otimes I_{\infty}$ satisfies the assertion by Theorem 5.3.

Acknowledgements. Research of both authors was supported by NSF. Li was also supported by the William and Mary Plumeri Award; he is an honorary professor of the University of Hong Kong and an honorary professor of the Taiyuan University of Technology.

\section{References}

[1] Y. S. Abramov, On the geometric properties of the joint spectrum of a family of self-adjoint operators, Studia Math. 61 (1977), 55-62.

[2] J. A. Anderson, On compact perturbations of operators, Canad. J. Math. 26 (1974), $247-250$.

[3] S. C. Arora and R. Kumar, Joint essential numerical range, Glas. Mat. Ser. III 18 (38) (1983), 317-320.

[4] Y. H. Au-Yeung and Y. T. Poon, A remark on the convexity and positive definiteness concerning Hermitian matrices, Southeast Asian Bull. Math. 3 (1979), no. 2, 85-92.

[5] Y. H. Au-Yeung and N. K. Tsing, An extension of the Hausdorff-Toeplitz theorem on the numerical range, Proc. Amer. Math. Soc. 89 (1983), 215-218.

[6] M. Barraa, Essential numerical range of elementary operators, Proc. Amer. Math. Soc. 133 (2005), 1723-1726.

[7] M. Barraa and V. Müller, On the essential numerical range, Acta Sci. Math. (Szeged) 71 (2005), 285-298.

[8] S. K. Berberian and G. H. Orland, On the closure of the numerical range of an operator, Proc. Amer. Math. Soc. 18 (1967), 499-503.

[9] P. Binding and C. K. Li, Joint ranges of Hermitian matrices and simultaneous diagonalization, Linear Algebra Appl. 151 (1991), 157-168.

[10] F. F. Bonsall and J. Duncan, Numerical Ranges of Operators on Normed Spaces and of Elements of Normed Algebras, London Math. Soc. Lecture Note Ser. 2, Cambridge Univ. Press, Cambridge, 1971.

[11] —, - Numerical Ranges, II, London Math. Soc. Lecture Note Ser. 10, Cambridge Univ. Press, Cambridge, 1973.

[12] J. J. Buoni and B. L. Wadhwa, On joint numerical ranges, Pacific J. Math. 77 (1978), 303-306.

[13] G. F. Cao, On the joint essential spectrum, the joint essential numerical range and the boundary of joint numerical range, J. Math. Res. Exposition 10 (1990), 25-31.

[14] M. Chō and M. Takaguchi, Boundary points of joint numerical ranges, Pacific J. Math. 95 (1981), 27-35.

[15] A. T. Dash, Joint numerical range, Glas. Mat. 7 (1972), 75-81.

[16] —, Joint spectra, Studia Math. 45 (1973), 225-237.

[17] -, Joint spectrum in the Calkin algebra, Bull. Amer. Math. Soc. 81 (1975), 10831085 .

[18] T. M. El-Adawy, M. Z. Abd-Alla and H. A. Atia, On the joint essential and joint maximal essential centre valued range, Proc. Math. Phys. Soc. Egypt 77 (2002), $29-49$. 
[19] M. K. H. Fan and A. L. Tits, On the generalized numerical range, Linear Multilinear Algebra 21 (1987), 313-320.

[20] P. Fan, C. K. Fong and D. A. Herrero, On zero-diagonal operators and traces, Proc. Amer. Math. Soc. 99 (1987), 445-451.

[21] P. A. Fillmore, J. G. Stampfli and J. P. Williams, On the essential numerical range, the essential spectrum, and a problem of Halmos, Acta Sci. Math. (Szeged) 33 (1972), 179-192.

[22] K. E. Gustafson and D. K. M. Rao, Numerical Range: The Field of Values of Linear Operators and Matrices, Springer, New York, 1997.

[23] E. Gutkin, E. A. Jonckheere and M. Karow, Convexity of the joint numerical range: topological and differential geometric viewpoints, Linear Algebra Appl. 376 (2004), 143-171.

[24] P. R. Halmos, A Hilbert Space Problem Book, 2nd ed., Grad. Texts in Math. 19, Encyclopedia Math. Appl. 17, Springer, New York, 1982.

[25] R. A. Horn and C. R. Johnson, Topics in Matrix Analysis, Cambridge Univ. Press, Cambridge, 1991.

[26] G. Ji, N. Liu and Z. Li, Essential numerical range and maximal numerical range of the Aluthge transform, Linear Multilinear Algebra 55 (2007), 315-322.

[27] M. S. Jones and H. P. Rogosinski, Inclusion relations involving the $k$-numerical range and the essential numerical range, ibid. 37 (1994), 161-173.

[28] S. P. Juneja, On extreme points of the joint numerical range of commuting normal operators, Pacific J. Math. 67 (1976), 473-476.

[29] J. S. Lancaster, The boundary of the numerical range, Proc. Amer. Math. Soc. 49 (1975), 393-398.

[30] D. A. Legg and D. W. Townsend, Essential numerical range in B( $\left.l_{1}\right)$, Proc. Amer. Math. Soc. 81 (1981), 541-545.

[31] C. K. Li and Y. T. Poon, Convexity of the joint numerical range, SIAM J. Matrix Anal. Appl. 21 (1999), 668-678.

[32] B. Magajna, On the essential numerical range of a generalized derivation, Proc. Amer. Math. Soc. 99 (1987), 86-92.

[33] Y. Ogata, Local distinguishability of quantum states in infinite-dimensional systems, J. Phys. A 39 (2006), 3059-3069.

[34] Y. T. Poon, On the convex hull of the multiform numerical range, Linear Multilinear Algebra 37 (1994), 221-223.

[35] —, Generalized numerical ranges, joint positive definiteness and multiple eigenvalues, Proc. Amer. Math. Soc. 125 (1997), 1625-1634.

[36] M. Takaguchi and M. Chō, The joint numerical range and the joint essential numerical range, Sci. Rep. Hirosaki Univ. 27 (1980), 6-8.

[37] J. P. Williams, The numerical range and the essential numerical range, Proc. Amer. Math. Soc. 66 (1977), 185-186.

Department of Mathematics

The College of William and Mary

Williamsburg, VA 23185, U.S.A.

E-mail: ckli@math.wm.edu
Department of Mathematics Iowa State University Ames, IA 50011, U.S.A. E-mail: ytpoon@iastate.edu

Received May 1, 2008 